\title{
Sufrimiento, estados afectivos y dolor
}

\author{
Suffering, affective states and pain
}

Efraín Gayosso Cabello

Universidad Nacional Autónoma de México egayosso@sofia.filosoficas.unam.mx

\section{Resumen}

En este texto desarrollo la idea que el sufrimiento no es lo mismo que el dolor, no es una emoción y tampoco un estado de ánimo, pero sí es parte de la afectividad. Está constituido por estados afectivos tales como emociones, estados de ánimo y dolor. Pero estos estados no son necesarios para que haya sufrimiento. Estos estados a veces son constitutivos del sufrimiento, pero cuando hay sufrimiento al menos un estado afectivo de este tipo es constitutivo del sufrimiento. Además, señalo que el sufrimiento a veces es una disposición y aclaro en qué sentido lo es. Las causas 
del sufrimiento pueden ser diversas, pero me centro en dos que considero son importantes para entender al sufrimiento, estas son la enfermedad y la pérdida. Al final argumento que se puede sufrir sin dolor y tener dolor sin sufrimiento, y que rara vez solamente el dolor es constitutivo del sufrimiento.

Palabras clave: Sufrimiento; emociones; dolor; enfermedad; narrativa autobiográfica; rasgos de carácter.

\section{Abstract}

In this text I develop the idea that suffering is not the same as pain, it is not an emotion and neither a mood, but it is part of affectivity. It is constituted by effective states such as emotions, moods and pain. But these states are not necessary in order to there be suffering. These states are sometimes constitutive of suffering, but when there is suffering at least one affective state of this type is constitutive of suffering. In addition, I point out that suffering is sometimes a disposition and I clarify in what sense it is so. The causes of suffering can be diverse, but I focus on two that I think are important for understanding suffering, these are illness and loss. In the end I argue that one can suffer without pain and have pain without suffering, and that only pain is rarely constitutive of suffering.

Key words: Suffering; emotions; pain; illness; autobiographic narrative; character trait. 


\section{Introducción}

A continuación, sostendré que el sufrimiento está constituido por estados afectivos. Además, explicaré que el sufrimiento no sólo está constituido por estados afectivos, sino que también pude ser una disposición a sentir ciertas emociones. Después, diré que el sufrimiento está relacionado con aspectos de la vida y estos aspectos son causa de sufrimiento. En especial me centraré en la noción de pérdida y la enfermedad. Al final argumentaré que para sufrir no es necesario sentir dolor, como que sentir dolor no implica necesariamente sufrir.

Cabe aclarar que la discusión que desarrollaré a continuación es filosófica, es decir, pretendo desarrollar argumentos que puedan sostenerse a priori. Sin embargo, en algunas partes me apoyaré en evidencia empírica o recurriré a casos dados por la literatura para sostener los argumentos. Además, un objetivo de lo desarrollado aquí es dar mayor claridad a lo que es el sufrimiento y pueda ser útil para otras disciplinas como la medicina.

\section{Estados afectivos y sufrimiento}

Algo que es característico del sufrimiento es que éste se siente mal, es decir, es desagradable sufrir. Independientemente de si se sufre por sentir un dolor crónico, un estado de ánimo o una emoción, siempre que se 
esté sufriendo será desagradable. Se puede decir que un componente esencial del sufrimiento es un afecto negativo (Brady 2018, 16). Así, las emociones, estados de ánimo y el dolor cuando implica sufrimiento ${ }^{1}$, son constitutivos del sufrimiento.

Un elemento que subyace a lo anterior es que aquellos estados tienen una fenomenología, es decir, las personas los sienten de algún modo. Al menos en estos estados, independientemente del modo característico en que se sientan, algo que es común a ellos es que se sienten de manera no placentera ${ }^{2}$. Esto último asocia estos estados con el sufrimiento, ya que sentir este tipo de emociones representa un estado de insatisfacción y no placer para las personas (ver Brady 2015).

En este punto cabe aclarar que sentir un estado afectivo no placentero o de insatisfacción no siempre implica que estos sean constitutivos del sufrimiento. A menudo estos estados son constitutivos del sufrimiento, como por ejemplo cuando una persona siente tristeza por la muerte de un amigo cercano. No obstante, hay veces en las que, aunque se sienta un estado de insatisfacción, éste no constituye

${ }^{1}$ El dolor, a pesar de que a menudo es desagradable experimentarlo, no siempre implica sufrimiento. Al final de este texto explicaré cuándo el dolor implica sufrimiento y cuándo no.

2 Aunque a veces el dolor también se siente de manera no placentera, lo no placentero no es algo esencial al dolor. De ahí que a veces implique sufrimiento y a veces no. Lo que es esencial al dolor es lo doloroso (painfulness) (Brady 2015, 403). 
sufrimiento. Por ejemplo, cuando una persona siente aburrimiento. Otro caso en el cual sentir una emoción desagradable no constituye sufrimiento es cuando las personas disfrutan de sentir emociones desagradables y buscan experimentarlas. Un ejemplo de esto es cuando las personas asisten a una mansión embrujada en un parque de diversiones para sentir miedo.

De lo anterior surge la pregunta de por qué no todos los afectos negativos constituyen sufrimiento. Se pueden dar al menos dos respuestas, una que contesta a la cuestión de por qué estados afectivos como el aburrimiento no constituyen sufrimiento y otra que contesta a la de por qué emociones que las personas buscan experimentar tampoco son parte del sufrimiento.

Estados afectivos como el aburrimiento no son parte del sufrimiento, porque en estos casos entran en juego elementos relevantes para estos estados. Estos elementos son la intensidad con que se sienten, así como pensamientos acerca de la relevancia de los objetos por los que surgen. Sentirse aburrido una tarde de domingo $y$, a la vez, sin ganas de hacer algo para ya no sentirse aburrido no es parte del sufrimiento, ya que no es un estado intenso. En cuanto a la relevancia, sentirse aburrido una tarde de domingo, aunque pueda ser desagradable, no es relevante en tanto pueda afectar a una persona más allá de pasar una tarde aburrida. Así, para que un estado afectivo sea parte del sufrimiento debe tener una intensidad que impacte en la persona y el objeto por el cual 
se tiene el estado debe ser relevante para la persona (Brady 2018, 30).

Para el segundo caso, una respuesta es que la persona que busca sentir miedo lo hace de manera voluntaria. En este sentido se puede decir que cuando una persona busca de manera voluntaria experimentar un estado desagradable, este estado no es parte del sufrimiento. Sin embargo, puede haber casos en los que las personas tienen involuntariamente experiencias que les causan estados afectivos desagradables y aun así no sufrir (ver Stump 2010, 11). Por ejemplo, un niño a quien obligan ir a la escuela y mientras está ahí siente hastío y preferiría estar en casa jugando videojuegos. Sobre él no se diría que sufre, a pesar de que involuntariamente va a la escuela y siente hastío por ello. El hastío que siente es desagradable para él, pero ir a la escuela es un beneficio. Se puede plantear que el niño ahora es adulto y piensa en retrospectiva cuánto odiaba ir a la escuela y el hastío que sentía, y ahora pensar que esos afectos ya no importan, y fue bueno para él que lo obligaran a ir a la escuela.

Otra manera de ver cómo las emociones son constitutivas del sufrimiento es pensándolas en términos del episodio emocional, emociones a corto y largo plazo, y disposiciones. De esta manera se puede ver cuándo un episodio emocional constituye al sufrimiento, cuándo una persona sufre al tener una emoción de largo plazo y cómo el sufrimiento puede disponer a sentir ciertas emociones. En este último punto no hay una emoción constitutiva del 
sufrimiento, sino que el sufrimiento dispone a sentir ciertas emociones.

El episodio emocional es cuando se siente la emoción en el momento y el episodio está acompañado por cambios corporales. Por ejemplo, cuando un profesor monta en cólera por una mala pregunta de su estudiante y enrojece. Las emociones de corto y largo plazo tienen que ver con un estado de la persona en el tiempo, y este tipo de emociones no están acompañadas por cambios corporales en el tiempo que duran. Una emoción de corto plazo, por ejemplo, es cuando alguien estuvo alegre durante su visita al parque de diversiones; mientras que una emoción de largo plazo es cuando alguien tiene resentimiento con sus padres durante años. Finalmente, las disposiciones tienen que ver con la disposición a sentir una emoción. Por ejemplo, tener miedo a las alturas. En este caso no es que se tenga el episodio emocional del miedo o que tenga una duración. El miedo a las alturas es la disposición a sentir miedo cuando se está en un lugar alto (Hansberg 1996, 13).

Según lo que constituya el sufrimiento, éste será episódico, tendrá una duración corta o larga. En estos tres casos se sigue manteniendo la característica no placentera del sufrimiento. Sin embargo, la fenomenología sobresaliente de lo desagradable sólo se da en el episódico, ya que en las emociones de corto o largo plazo no hay una fenomenología persistente. 
Como se ha dicho, cuando se tiene un episodio emocional se sufre si la emoción que se está sintiendo tiene una sensación desagradable y el objeto al que se dirige es relevante para el sujeto. En este caso, el episodio emocional constituye al sufrimiento, y éste dura lo que dura el episodio emocional. Por lo general, la duración de los episodios emocionales es muy breve. Esto se debe a que los episodios emocionales requieren mucha energía del organismo por lo que la tendencia es que tengan una duración breve (Damasio 2010, 246).

En cuanto a las emociones de corto y largo plazo, según sea la emoción que constituya al sufrimiento, éste será de corto o largo plazo. Se sufre a corto plazo, por ejemplo, cuando alguien, después de unos minutos de esperar a su cita, comienza a sentir desesperación. En cambio, alguien sufre a largo plazo cuando, por ejemplo, una persona ha trabajado algunos años en el mismo lugar y durante el tiempo que ha trabajado ahí ha sentido envidia porque cree que sus compañeros tienen beneficios laborales inmerecidamente, mientras que él no ha obtenido nada.

Lo dicho anteriormente fue en cuanto al sufrimiento constituido por emociones ${ }^{3}$. Pero, además de lo anterior, el sufrimiento en ocasiones también es una disposición. Si bien en los casos anteriores sufrir se siente de algún modo, ya sea

${ }^{3}$ Más adelante explicaré cuándo el sufrimiento está constituido por estados afectivos que no son emociones. En especial me centraré en estados de ánimo. 
porque se tienen ciertas emociones o se siente un episodio emocional, también el sufrimiento puede disponer a tener algunas emociones. Por ejemplo, cuando una persona sufre por discriminación ${ }^{4}$, esta persona que está sufriendo puede tener la disposición a sentir impotencia, enojo, resentimiento hacia aquellas personas que la discriminan, tristeza, vergüenza, etcétera.

Aquí cabe hacer una distinción más sobre las disposiciones. Las disposiciones pueden ser single-track y multi-track (Deonna y Teroni 2012, 8). Las single-track son sobre una emoción, como cuando alguien teme a las alturas. En este caso, la persona que le teme a las alturas tiene la disposición a sentir miedo. Mientras, las disposiciones multitrack son sobre varias emociones. Ejemplos de estas disposiciones son el amor y el odio. Cuando una persona está enamorada está dispuesta a sentir varias emociones como orgullo por los logros de la persona que ama, admiración, pena si no es correspondida o celos si aparece algún rival.

Así, respecto al sufrimiento, cuando éste es una disposición parece ser que es una del tipo multi-track, porque el sufrimiento dispone a sentir varias emociones y no a sentir una emoción en particular. Las emociones a las que el sufrimiento puede disponer son diversas y aquí no me detendré a decir cuáles son. Estas emociones a las que

${ }^{4}$ En este caso la discriminación es causa del sufrimiento. Más adelante abordaré cómo entender las causas del sufrimiento. 
dispone el sufrimiento dependerán del contexto en el que el sufrimiento sea causado.

Con lo dicho hasta aquí he tratado de aclarar cómo el sufrimiento está constituido por emociones, cuándo se puede hablar de sufrimiento, cuándo se tiene una emoción desagradable y cuándo, a pesar de sentir una emoción desagradable, no hay sufrimiento. También he tratado de aclarar que el sufrimiento a veces es una disposición a sentir emociones.

Ahora falta aclarar que no sólo las emociones son constitutivas del sufrimiento. También se puede sufrir al sentir dolor o al tener un estado de ánimo. La relación entre dolor y sufrimiento la abordaré más adelante. En lo que sigue me ocuparé de aclarar en qué sentido los estados de ánimo son constitutivos del sufrimiento.

Una intuición que se tiene es que las emociones y los estados de ánimo son estados afectivos distintos. Mientras las emociones por lo general son estados breves, pensando en este sentido en el episodio emocional, los estados de ánimo son más duraderos. Sin embargo, también hay emociones a largo plazo, por lo que distinguirlas de los estados de ánimo por su duración no es adecuado.

Por otro lado, los estados de ánimo y las emociones se pueden distinguir en cuanto al objeto al que se dirigen. Las emociones se dirigen a objetos específicos como cuando alguien tiene miedo a la araña que va bajando del techo. Mientras que los estados de ánimo no se dirigen a un objeto 
especifico, su objeto es menos claro. Una metáfora que se utiliza para capturar la noción de que el objeto al que los estados de ánimo se dirigen es más general o menos claro, es que los estados de ánimo se dirigen al mundo coloreándolo. Así, cuando una persona está deprimida, su depresión se dirige al mundo como coloreándolo de cierta manera que ve todo como desprovisto de valor (Deonna y Teroni 2012, 105106; Goldie 2000; 143-151).

Aunque lo anterior es controversial, no me detendré más en discutir cómo distinguir los estados de ánimo de las emociones. Para los intereses de este texto basta con entender que los estados de ánimo son distintos a las emociones, y en cuanto que se sienten de algún modo y colorean al mundo de cierta manera son constitutivos del sufrimiento.

Cuando se está en un estado de ánimo como la depresión, las personas sufren porque es un estado desagradable y para la persona deprimida nada es satisfactorio. Las personas con depresión sufren al estar en ese estado, porque además de cómo se siente la depresión, también las lleva a ver al mundo como desprovisto de valor, y sufren al sentir que no vale la pena seguir viviendo. En este sentido, no sólo la depresión es constitutiva del sufrimiento de la persona deprimida, también dispone a que esta persona vea al mundo desprovisto de valor y llevarla a pensar que nada vale la pena.

Hasta aquí he planteado cómo el sufrimiento está constituido por emociones y estados de ánimo. Cabe aclarar 
que estos estados afectivos no son necesarios para que haya sufrimiento. A veces las personas sufren al sentir una emoción o al tener un estado de ánimo. También las personas a veces sufren al sentir emociones a la vez que tienen un estado de ánimo. Estos estados no son excluyentes entre sí, como tampoco son necesarios para el sufrimiento: a veces hay emociones, a veces estados de ánimo o a veces ambos.

El sufrimiento no es una emoción o un estado de ánimo, sino que es un estado afectivo constituido a veces por emociones o a veces por estados de ánimo. También el sufrimiento puede ser una disposición multi-track al disponer a sentir ciertas emociones. El sufrimiento no se reduce a las emociones ni a los estados de ánimo. A continuación abordaré algunas causas del sufrimiento y algunas relaciones que tiene con las personas.

\section{Causas del sufrimiento y aspectos de la persona}

El sufrimiento está relacionado con distintos aspectos de la vida humana. Para Eric J. Cassell (2004, 42-44) el sufrimiento tiene que ver con la personalidad, i.e., con cómo las personas viven su sufrimiento y cómo lo superan. Con la pérdida, la cual debe ser entendida de manera amplia, ya que se puede sufrir por la pérdida de un ser querido, por perder el patrimonio o por la pérdida de capacidades. Otro aspecto relacionado con el sufrimiento es el de los planes o aspectos relacionados con el pasado y el futuro. Las personas sufren 
porque sus planes se ven frustrados, o porque imaginan algún obstáculo que pudiera frustrar lo planeado. También las personas pueden sufrir por algo sucedido en el pasado. Por último, otro aspecto relevante para el sufrimiento es el de la enfermedad. Las personas sufren cuando están enfermas, pero no sólo sufren por los padecimientos propios de la enfermedad, hay otros aspectos que la enfermedad afecta y que son causa de sufrimiento.

En lo que sigue abordaré primero al menos dos causas del sufrimiento: la pérdida y la enfermedad. Aunque puede haber diversas causas del sufrimiento, me centraré en estas dos porque capturan bien varias intuiciones que se tienen sobre el sufrimiento. Después hablaré sobre cómo se relaciona el sufrimiento con la personalidad y rasgos de carácter. Finalmente desarrollaré una explicación narrativa del sufrimiento donde plantaré cómo opera el sufrimiento sobre el pasado, el futuro y situaciones contrafácticas.

\section{a) Pérdida y sufrimiento}

Las pérdidas pueden causar sufrimiento de manera importante y están relacionadas con diferentes aspectos de la vida humana. Se sufre ${ }^{5}$ cuando muere un ser querido, pero también se sufre cuando se pierden capacidades a causa de

${ }^{5}$ Aunque las pérdidas pueden causar distintas emociones, hablaré en términos de sufrimiento dado que está constituido por las emociones causadas por las pérdidas. 
una enfermedad o hay pérdidas materiales a causa de un desastre natural. En el caso de la muerte de un ser querido se sufre porque es una pérdida irreparable.

En el caso de pérdidas materiales, éstas podrían recuperarse o no, y aunque se puedan recuperar, las personas pueden sufrir si recuperarlas representa mucho esfuerzo. Por ejemplo, una persona que pierde su casa a causa de un terremoto sufre porque posiblemente era su único patrimonio, no tiene a donde ir después de la pérdida de su casa y no tiene recursos económicos para rentar una casa o reconstruir la casa que ha perdido.

Además, la persona que sufre por la pérdida de su casa también puede sufrir por otros aspectos relacionados a este suceso. Esta persona puede desarrollar estrés postraumático por el terremoto, sufrir por alguna lesión causada por el derrumbe de su casa, sufrir por la falta de víveres o de asistencia después del terremoto, etc. En este sentido, la causa del sufrimiento no se reduce a la pérdida, sino que también tiene que ver con otros aspectos que se dan en el contexto de la pérdida.

En lo anterior se pueden ver ejemplos de aquello que es constitutivo del sufrimiento y de aquello que lo causa. La pérdida material, el terremoto, la pérdida de alguna capacidad física por lesión y la falta de víveres son ejemplos de causas del sufrimiento. Mientras que la ansiedad generada por la pérdida material, el estrés post traumático generado por el terremoto, la impotencia de no poder 
moverse por incapacidad física y el hambre por falta de víveres son constitutivos del sufrimiento.

Otro tipo de pérdida por la que se puede sufrir es la pérdida de capacidades. Las causas de la pérdida de capacidades son diversas. Se pueden perder capacidades por una enfermedad, por un accidente o por la edad. En estos tres aspectos se puede identificar que principalmente se sufre por la pérdida de la capacidad de ser autónomo y necesitar de la ayuda de alguien más para realizar tareas simples como caminar. Aunque también puede haber pérdida de capacidades cognitivas. Estas personas, ya sea por una enfermedad, por un accidente o por tener edad avanzada, dejan de valerse por sí mismas y necesitan el cuidado de alguien más. También la pérdida de capacidades puede causar sufrimiento en aquellos que se encargan de su cuidado, ya que el cuidar de estas personas puede causar modificaciones en la vida de los cuidadores no profesionales quienes por lo general son familiares.

\section{b) Salud y sufrimiento}

La enfermedad es una causa importante de sufrimiento. Las personas sufren cuando caen enfermas debido a los padecimientos propios de la enfermedad. Por ejemplo, cuando una persona enferma de cáncer de huesos sufre a causa de los dolores insoportables propios de la enfermedad. También cuando enferman las personas sufren por la 
pérdida de capacidades causada por la enfermedad. Una persona enferma de Alzheimer sufre por la pérdida de sus capacidades cognitivas al ser incapaz de recordar o reconocer personas cercanas a ella como reconocer a sus hijos. Una persona con glaucoma también puede sufrir al perder la capacidad de visión.

Sin embargo, la persona enferma no sólo sufre por los padecimientos de su enfermedad, también puede sufrir por otras circunstancias relacionadas con su enfermedad. En países donde el sistema de salud es deficiente, además de que las personas sufren por el padecimiento, también sufren por recibir una atención deficiente.

Además, un sistema de salud deficiente también puede causar sufrimiento en el personal de salud. Ellos pueden sufrir por trabajar en condiciones desfavorables como no contar con el equipo necesario para atender a los pacientes. También pueden sufrir estrés $\mathrm{u}$ otros desordenes causados por la sobrecarga de trabajo resultado de la saturación del sistema de salud y la falta de infraestructura para atender la sobrepoblación de pacientes.

Por otra parte, las personas externas al sistema de salud, pero relacionadas con el paciente como pueden ser familiares o amigos, también sufren a causa de la enfermedad. Las personas cercanas sufren ya sea porque tienen empatía con el paciente al ver su sufrimiento o sufren porque se vuelven cuidadores del paciente y en este sentido deben modificar algunos hábitos. Así, otros sufren por lo que le pasa al 
paciente, y en el caso del cuidador cuando este por lo general es un familiar y no un cuidador profesional se ve afectado directamente al tener que modificar su ritmo de vida.

Con esto no intento limitar al sufrimiento a solo la pérdida y la enfermedad. Aquí me he centrado en esas causas porque capturan varios aspectos que también son causa del sufrimiento. A partir de su análisis se puede ver la diferencia entre lo que causa y lo que es constitutivo del sufrimiento. Así, el sufrimiento no se puede reducir a una causa, sino que tiene causas variadas.

\section{c) Personalidad y sufrimiento}

La manera en que se experimenta el sufrimiento y es superado puede variar de persona a persona. Por ejemplo, ante el fallecimiento de un ser querido, una persona aprensiva sufrirá más que una persona que no lo es, aunque ambas personas estén sufriendo. También, la persona aprensiva tardará más en superar su sufrimiento por el fallecimiento que una persona que no lo es. Esto se debe a la capacidad de resiliencia que tienen las personas y la cual está estrechamente relacionada con el sufrimiento.

La resiliencia es la capacidad de superar al sufrimiento. Una persona que ha desarrollado mejor esta capacidad puede superar más fácil algún suceso traumático que otra que no haya desarrollado esta capacidad. Así, de un suceso traumático como la muerte de un ser querido, una persona 
con mejor resiliencia puede superar el sufrimiento causado por este suceso sin más complicaciones, mientras que a otra persona que no haya desarrollado esta capacidad le costará más trabajo superar su sufrimiento (Krashin, Murinova, Howe y Ballantyne 2014, 113).

El desarrollo de esta capacidad depende del entorno en el cual las personas crecieron o están insertas al momento en que el suceso que les causa sufrimiento se presentó. Los principales factores que intervienen en el desarrollo de la resiliencia son la familia, la educación y la cultura (Fleming y Ledogar 2008, 8). La familia tiene que ver con la resiliencia, porque es el primer círculo social en el que las personas se desarrollan. En este aspecto, la resiliencia pude ser desarrollada mejor en una familia que no es disfuncional, esto es, donde los conflictos, la mala conducta o los abusos no están presentes. La educación también es importante, ya que a través de ésta se puede orientar a las personas sobre cómo superar al sufrimiento.

Otro aspecto que tiene que ver con aspectos de la persona y su relación con el sufrimiento son los rasgos de carácter. Antes de continuar con la relación entre rasgos de carácter y sufrimiento haré algunas aclaraciones respecto a los rasgos de carácter. La palabra «carácter» es derivada del griego kharaktér, que se refiere a la marca que se ponía sobre las monedas o al hierro para marcar ganado. De ahí que el sentido de «carácter» sea aquello que distingue de lo demás. En el uso contemporáneo de la palabra se ha llegado a usar 
como el conjunto de cualidades que distingue a un individuo de otro. Así, en el uso contemporáneo de la palabra «carácter» se llega a utilizar como sinónimo de «personalidad» (ver Homiak 2016).

Sin embargo, en filosofía el término «carácter» tiene otras connotaciones. Aristóteles en la Ética Nicomáquea (1985, 1103a) dice que hay dos clases diferentes de excelencias humanas: excelencias de pensamiento y excelencias de carácter. Cuando se habla de excelencias del carácter, esto se ha tendido a relacionar con virtudes. Así, el carácter no sólo tiene que ver con cualidades de individualidad o personalidad, sino que tiene que ver con las cualidades del individuo que hacen que sea una persona admirable. En contraposición a las virtudes están los vicios. Bajo esta concepción, una persona no virtuosa o viciosa es aquella con rasgos de carácter contrarios a la excelencia.

Se han hecho críticas a la idea de virtudes y rasgos de carácter desde el situacionismo. Para esta postura los rasgos de carácter no son robustos o globales, lo cual significa que no son estables o consistentes a través de distintas situaciones, y es erróneo invocarlos para explicar las acciones de las personas. Para apoyar su postura, los filósofos que argumentan a favor del situacionismo se apoyan en evidencia dada por experimentos en psicología social, que muestran que «gran parte del comportamiento humano es atribuible a rasgos aparentemente triviales de las situaciones 
en que las personas se encuentran $»^{6}$ (Homiak 2016, parte 5.1). Así, para el situacionismo no hay rasgos de carácter que expliquen el comportamiento de las personas de manera global, sino que el comportamiento se explica a través de rasgos que surgen según la situación en la que se encuentre la persona 7 .

Una respuesta al situacionismo es dada por el modelo del «sistema de la personalidad cognitivo-afectiva» (CAPS por sus siglas en inglés). Este modelo se apoya también en estudios en psicología. Por ejemplo, para desarrollar su teoría, Snow (2010) se basa en estudios sobre la activación de estereotipos y la inhibición de la conducta, así como en estudios sobre la automaticidad de la dependencia de fines. En estos estudios se muestra que la repetición de metas o fines influyen en la conducta.

Así, para el CAPS los rasgos de carácter «son paquetes integrados por motivaciones distintivas, cogniciones $\mathrm{y}$ elementos afectivos, en los cuales los componentes cognitivos $\mathrm{y}$ afectivos son formados $\mathrm{y}$ dirigidos por motivaciones características de la virtud» (Snow 2015, 366). De acuerdo con Snow las virtudes son subconjuntos de los rasgos de carácter los cuales son locales, pero pueden llegar a ser globales a través del hábito.

${ }^{6}$ Las traducciones son mías, a menos que se cite la traducción al español cuando haya una disponible.

7 Posturas situacionistas se pueden encontrar en Harman (1999; 2000), Doris (2002), Vranas (2005). 
Otra propuesta que parte del modelo de CAPS es dada por Russell (2009). Para él, el modelo de CAPS es una teoría de la personalidad y define "carácter» como la parte de la personalidad que tiene significado normativo. En su teoría los rasgos de carácter se entienden como un subconjunto de la personalidad con relevancia ética o también se pueden entender como el todo de la personalidad desde un punto de vista ético. De ahí su importancia para la conformación de virtudes.

Bajo esta perspectiva es posible considerar los rasgos de carácter como un subconjunto de aquello que constituye la personalidad y que están estrechamente relacionados con las virtudes. Tanto para Russell como para Snow el modelo de CAPS es una teoría de la personalidad que toma en cuenta los rasgos de carácter. A pesar de que pueda haber evidencia en psicología social de que no son globales ni estables, hay evidencia en estudios psicológicos a favor de que hay rasgos de carácter que, si bien son locales, pueden llegar a ser globales a través del hábito y su ejercitación.

Ahora bien, en cuanto al sufrimiento, los rasgos de carácter influyen en la forma en que las personas se relacionan con éste. Una persona optimista tendrá una mejor disposición para superar algún suceso que sea causa de sufrimiento, mientras que una persona pesimista tendrá la disposición a sufrir más que la persona optimista.

Puede haber otros rasgos de carácter relacionados con el sufrimiento como ser cruel, afable, empático, generoso, 
apático, egoísta, etc. Por ejemplo, la persona cruel tendrá la disposición a regocijarse ante el sufrimiento de los demás, la egoísta a no prestarle atención al sufrimiento de los demás mientras ella no sufra y la apática a no prestarle atención al sufrimiento de otros. En cambio, las personas afables, generosas y empáticas tendrán la disposición a ver el sufrimiento de los demás como algo que se tiene que aliviar.

En este sentido, siguiendo una teoría de CAPS, los rasgos de carácter son subconjuntos de la personalidad que pueden ser ejercitados por el hábito y llegar a ser virtudes respecto al sufrimiento. Así, las personas con rasgos de carácter tales como la crueldad o el egoísmo tienden al vicio, mientras que las personas con rasgos como la amabilidad tienden a la virtud en tanto que son sensibles al sufrimiento de los demás.

\section{d) Narración y sufrimiento}

Recupero la noción de narración y la de emociones de Peter Goldie (2012). En esta concepción el pensamiento narrativo tiene una estructura interna y una externa. En la estructura interna la persona es protagonista. Por ejemplo, cuando una persona está esperando a alguien más en la estación del tren para partir juntos en el tren de las 12:30, y la persona a la que está esperando no llega faltando dos minutos para que el tren parta, la persona que espera puede comenzar a imaginar una historia en la cual la persona que 
está esperando no llega y ella tiene que partir sin él sintiéndose indignada porque no llegó (ver Goldie 2012, 77).

En la estructura externa la persona tiene la perspectiva del narrador o de la audiencia, y en este sentido tiene emociones acerca de la narración, pero no como si fuera su protagonista. Por ejemplo, cuando la misma persona del ejemplo anterior comienza a creer que la persona a la que está esperando no llegará a tiempo porque faltan dos minutos para que el tren parta, en lugar de imaginar que se va sin la persona a la que espera comienza a imaginar que a quien espera no llegará a tiempo porque ha sufrido un accidente.

En los ejemplos anteriores las narraciones van hacia el futuro, pero en el pensamiento narrativo también se pueden estructurar historias sobre sucesos contrafácticos. Un ejemplo es cuando alguien que ha perdido un avión por haber hecho sus maletas a última hora se arrepiente de no haberlas hecho un día antes. Esta persona estructura una historia en la que hace sus maletas un día antes, llega a tiempo al aeropuerto y no pierde su avión. Para Goldie (2012, 87), este tipo de narraciones y el arrepentimiento pueden tener un efecto de retroalimentación en el sentido de que la persona puede aprender de sus errores. Siempre que esta persona recuerde la historia de haber perdido su avión por no hacer sus maletas con tiempo y el arrepentimiento que sintió, será más precavida a la hora de tomarse el tiempo para preparar sus maletas. También los pensamientos contrafácticos pueden operar como frenos para hacer algo. 
Por ejemplo, cuando un político, por pensar en el sufrimiento que puede causar en una localidad, desiste de promulgar una ley que la afectaría.

Además de tener pensamientos narrativos sobre el futuro y sobre sucesos contrafácticos, las personas tienen pensamientos narrativos sobre el pasado. Al igual que el pensamiento narrativo futuro, el pasado puede tener una estructura interna o externa. En el caso de narraciones pasadas, la estructura es interna cuando la narración tiene significado para la persona como parte de ésta. Por ejemplo, cuando alguien recuerda haber golpeado su pastel de cumpleaños cuando cumplió seis años, porque no era del sabor que esperaba, y siente vergüenza al pensar en ese suceso. En cambio, la estructura es externa cuando la persona tiene el papel de narrador y no de personaje de la historia (Goldie 2012, 26).

Esta estructura sobre el pensamiento narrativo también puede ser pensada sobre el sufrimiento. Las personas a menudo sufren por cosas pasadas, por el futuro o por pensamientos contrafácticos del tipo «si sólo hubiera hecho $x$, no hubiera pasado $y »$. A continuación daré algunos ejemplos en los que las personas sufren por narraciones pasadas, futuras y contrafácticas. Al final también plantearé que la narración autobiográfica es necesaria para el sufrimiento.

Las personas sufren al imaginar alguna historia en la que no se cumple lo planeado o hay una situación difícil de 
superar para lograr lo planeado. Por ejemplo, cuando una persona, después de hacerse unos análisis médicos debido a un dolor que siente en el pecho, construye una historia en la que los análisis dan positivo a una enfermedad mortal. Esta persona piensa que sucederá lo peor y comienza a sentir ansiedad, preocupación y otras emociones asociadas al resultado que podría salir de los análisis. Ella sufre como resultado de la historia que se ha construido sobre lo que podría pasar en el futuro con su salud.

También se pude pensar en casos en que la persona sufre por una historia hacia el futuro donde no es protagonista. Por ejemplo, cuando una persona imagina con alguien más a su pareja durante el viaje que ésta realizará dentro de algunos días. En este caso, la persona puede sentir celos, desconfianza y otras emociones relacionadas con la historia que ha imaginado. La persona sufre al no sentirse a gusto sobre el viaje que realizará su pareja.

Los casos de narraciones contrafácticas pueden ser causa de sufrimiento muy a menudo. Por lo general, estas narraciones tienen que ver con resultados negativos consecuencia de actos en el pasado en los que no hubo previsión del resultado que se tendría. Así, estas narraciones tienen la estructura: «si no hubiera hecho $x$ no hubiera pasado $y » 0$ «si hubiera hecho $w$, hubiera pasado $z »$. Una persona que se arrepiente por no haber hecho con tiempo su equipaje sufre al sentir arrepentimiento. También en las narraciones contrafácticas se puede prever el sufrimiento que 
se pueda tener y así desistir de hacer algo. En este caso la estructura es: «si hago $x$, podría pasar $y$; $y$ puede causar sufrimiento, por lo que es mejor no hacer $x »$.

Las personas también sufren por cosas pasadas. En este caso puede haber narraciones del pasado en las que hay algo que hace sufrir a las personas. En la novela Job, de Joseph Roth (2017 [1930]), el protagonista es un judío ruso llamado Mendel que tiene tres hijos. El más pequeño de sus hijos se llama Menuchim y es epiléptico con un cierto grado de retraso mental. El hijo de en medio migra a Estados Unidos. Después de unos años, este hijo manda traer a su padre desde Rusia a Estados Unidos, y Mendel abandona a su hijo menor en Rusia. Mendel se establece en Estados Unidos y pasan los años, pero no olvida a Menuchim, y a pesar de los años sigue sufriendo por haber abandonado a su hijo. Cuando Mendel piensa en su hijo, esto puede tener una estructura interna o externa. Interna cuando recuerda el suceso de abandonar a su hijo y recuerda el sufrimiento que sintió. Externa cuando Mendel reconstruye la historia de su hijo como narrador a sus amigos con los que se reúne en Nueva York y les hace ver su sufrimiento por haber abandonado a su hijo.

Por último, la narración autobiográfica permite que las personas tengan una perspectiva de su sufrimiento: qué es lo que lo causó en el pasado o a dónde puede llegar, y así entender el sufrimiento que sienten. Un caso que muestra esto es el de las personas con amnesia retroactiva quienes 
sufren desde la perspectiva de la tercera persona, pero no desde su propia perspectiva.

Oliver Sacks (2009 [1985]) describe el caso de un paciente con este padecimiento. En 1975 Jimmie llegó al asilo donde trabajaba por entonces Sacks con una nota que decía «desvalido, demente, confuso y desorientado». Al empezar con el diagnostico de Jimmie, Sacks observó que presentaba una inteligencia normal, que no tenía problemas para socializar, era una persona dispuesta a colaborar, y que era amable y elocuente. En apariencia no mostraba ningún indicio de que sufriera. Sin embargo, Sacks empezó a identificar que había un problema cuando a preguntas sobre los planetas o sobre los elementos de la tabla periódica, Jimmie respondía con información no actualizada. Cuando le preguntó por su edad él respondió que tenía 19 años aunque claramente tenía más de 45. Así, Sacks descubrió que Jimmie padecía amnesia retroactiva. Su memoria se había quedado en 1961, y todo lo que había pasado desde entonces simplemente no era parte de Jimmie. Cada día que pasaba, Jimmie olvidaba que estaba en 1975 y su memoria sólo databa de 1961 hacia atrás. Por esta razón, él no sabía que sufría, para él todo estaba bien. Sólo las personas que lo rodeaban sabían que él sufría, y que había perdido 14 años de su vida. Sacks dice al respecto que «[l]o peculiar es que el horror sólo lo sienten los demás: el paciente, inconsciente, amnésico a su amnesia, puede seguir con lo que está haciendo, tan tranquilo, y no descubrir hasta después que 
perdió [...] media vida, y que no se dio cuenta» (Sacks 2009 [1985], 64). De esto se puede decir que la narración autobiográfica es necesaria para que en primera persona se tenga en cuenta que uno está sufriendo. Los demás ven el sufrimiento, pero si no se tiene una narración uno no ve el sufrimiento propio.

\section{Dolor y sufrimiento}

A menudo los conceptos de dolor y sufrimiento se utilizan como sinónimos. Esto se debe a que cuando una persona siente dolor se piensa que también está sufriendo. Lo anterior puede ser cierto en ciertas situaciones, sin embargo, el dolor no siempre implica sufrimiento y el sufrimiento no siempre está constituido por dolor.

El dolor, al igual que el sufrimiento, se siente de alguna forma, se sienten de manera desagradable. Debido a que se sienten de manera desagradable los organismos evitan el dolor o sufrir. Sin embargo, se puede pensar en situaciones en que, a pesar de la sensación desagradable del dolor, su experiencia se disfruta y no hay una motivación para evitarlo (Bain y Brady 2014, 2-3). Por ejemplo, comer algo picante o practicar el masoquismo son experiencias que causan dolor, pero algunas personas buscan comer alimentos picantes o practicar el masoquismo porque lo disfrutan a pesar de sentir dolor. En este sentido, el dolor se distancia del sufrimiento, 
porque las personas que buscan esas experiencias las disfrutan en lugar de sufrir.

En estos casos, aunque hay presencia de dolor, la característica de lo desagradable no está presente. En cambio, el sufrimiento siempre es desagradable. Se puede pensar en casos de personas de las que coloquialmente se dice que «les gusta sufrir». En estos casos, las personas en situaciones que les casusa sufrimiento no buscan evitarlas o cambiar la situación para dejar sufrir, e incluso hay personas que buscan situaciones que les causen sufrimiento. Estos casos son problemáticos porque suponen intenciones complejas de los agentes. Una respuesta que se puede dar es que en el primer caso la persona se autoengaña sobre situación. Para ella su situación no es causa de sufrimiento o a pesar de saberlo no es capaz de dejar de sentir la emoción que constituye a su sufrimiento ${ }^{8}$.

Otros casos en que el dolor no es constitutivo del sufrimiento, a pesar de que sí está presente el componente de lo desagradable, es cuando el dolor que se siente no es muy

\footnotetext{
${ }^{8}$ Este caso puede ser equiparable al de las emociones recalcitrantes. Una emoción es recalcitrante cuando una persona, a pesar de creer que no tiene por qué sentir la emoción y tener toda la evidencia a favor de sostener un juicio que vaya contrario a la emoción que siente, sigue sintiendo la emoción (ver Benbaji 2013; Brady 2009; D’Arms y Jacobson 2003; Tappolet 2012). Por ejemplo, una persona que se avergüenza por su etnia a pesar de saber que no hay superioridad entre etnias y que pertenecer a una $u$ otra no influye en las capacidades que uno como persona pueda desarrollar.
} 
intenso o no pasa más allá de un periodo corto. Un ejemplo es cuando uno se golpea la punta del dedo del pie con la esquina de un mueble. Aunque el dolor de pegarse en la punta del dedo puede ser muy intenso, es dudoso que este dolor sea constitutivo del sufrimiento.

Así como a veces el dolor no es constitutivo del sufrimiento, i.e., las personas no sufren cuando sienten dolor, no se puede negar que hay ocasiones en que el dolor es constitutivo del sufrimiento. Por ejemplo, cuando una persona enferma de cáncer de huesos no soporta el dolor causado por su enfermedad. En este caso el dolor que siente la persona enferma es constitutivo de su sufrimiento. Sin embargo, el dolor que siente el paciente no es lo único que constituye su sufrimiento. El caso del sufrimiento del paciente es más complejo, ya que también sufre al sentir emociones causadas por su situación, tener estados de ánimo como depresión por lo que su enfermedad significa para su vida, etc. Así, aunque el dolor a veces es constitutivo del sufrimiento, éste no es lo único que lo constituye.

Se puede pensar en casos menos complejos al del enfermo de cáncer en los que el dolor es constitutivo del sufrimiento: una persona con un dolor crónico como una migraña que puede durar días, semanas o meses. Sin embargo, al igual que con el enfermo de cáncer, la persona con migraña no sufre sólo al sentir el dolor. Hay otros estados que constituyen su sufrimiento como desesperación porque el dolor no cesa, impotencia porque no puede realizar sus 
tareas cotidianas o la fotosensibilidad causada por la migraña.

Con lo anterior se puede concluir que el dolor a veces es constitutivo del sufrimiento y cuando lo llega a ser no es el único estado que lo constituye, hay otros estados afectivos, además del dolor, que constituyen al sufrimiento. También se ha visto que el dolor no siempre implica que la persona esté sufriendo, puede sentir dolor sin implicar sufrimiento aun cuando la experiencia de dolor sea desagradable.

\section{Glosario}

Disposición afectiva: A diferencia de los estados afectivos, las disposiciones afectivas no tienen sensaciones. La disposición afectiva dispone a los sujetos a sentir estados afectivos cuando se dan las condiciones necesarias para que la disposición se actualice. Las disposiciones afectivas pueden dividirse en single-track y multi-track.

Disposición afectiva multi-track: Disposición relacionada con diversos estados afectivos. Ejemplos de disposición multi-track son el amor y el odio. Cuando se actualiza la disposición de 'amar' a estados afectivos se pueden sentir estados tales como celos, orgullo, excitación sexual, alegría o ansiedad (ver Deonna y Teroni 2012,8).

Disposición afectiva single-track: A diferencia de las disposiciones multi-track, estas disposiciones están relacionadas con sentir un único estado afectivo. Por 
ejemplo, tener miedo a las alturas. Cada vez que se actualice la disposición de tener miedo a las alturas el estado afectivo que se sentirá es miedo (ver Deonna y Teroni 2012, 8).

Dolor: Aquí dolor se entiende como dolor físico. El dolor es una sensación que pertenece a la clase de las sensaciones corporales como la picazón, la temperatura o los orgasmos. Las sensaciones corporales se caracterizan principalmente por ser localizadas en una parte específica del cuerpo. Cabe aclarar que la parte donde se identifica el dolor físico no siempre corresponde a la causa del dolor, es decir, se puede sentir dolor en una parte del cuerpo y la causa de ese dolor estar en otra parte. También, para sentir dolor en una parte específica no es necesario que tal parte de hecho sea parte del cuerpo, por ejemplo, el problema del miembro fantasma (ver Aydede 2019).

Emoción: Estado afectivo que se caracteriza por tener una sensación, una fenomenología, estar dirigida a un objeto en particular y una tendencia de acción que puede cumplirse o no. Por lo general, las emociones también incluyen cambios corporales como aceleración del pulso o aumento de la presión sanguínea (ver Goldie 2000, 12-13).

Estado afectivo: Estado mental que se caracteriza por tener una fenomenología, es decir, una experiencia particular y una sensación.

Fenomenología: Experiencia particular de un estado mental, ya sea un estado afectivo o un estado perceptual, como ver un color. 
Sensación: La capacidad de ser afectado por un estado afectivo (emoción, estado de ánimo, dolor, etcétera). El sentido en el que se utiliza aquí este término es parecido al término en inglés feeling.

\section{Bibliografía}

Aristóteles. (1985). Ética Nicomáquea, P. Palli Bonet (trad.). Gredos: Madrid.

Aydede, M. (2019). Pain. E. N. Zalta (ed.) Recuperado el 31 de octubre de 2019, de The Stanford Encyclopedia of Philosophy:

https://plato.stanford.edu/archives/spr2019/entries/pai $\mathrm{n} /$.

Bain, D. y Brady, M. S. (2014). Pain, pleasure and unpleasure. Review of Philosophy and Psychology, 5 (1), 1-14.

Benbaji, H. (2013). How is recalcitrant emotion possible? Australasian Journal of Philosophy, 91 (3), 577-599.

Brady, M. S. (2015). Seeing bad and feeling bad. Dialectica, 69 (3), 403-416.

Brady, M. S. (2018). Suffering and virtue. Oxford: Oxford University Press.

Brady, M. S. (2009). The irrationality of recalcitrant emotions. Philosophical Studies, 145 (3), 413-430.

Cassell, E. J. (2004). The nature of suffering and the goals of medicine (2a ed.). Nueva York: Oxford University Press. 
Damasio, A. (2010). Selfcomes to mind: constructing the conscius brain. Nueva York: Pantheon.

D'Arms, J., \& Jacobson, D. (2003). The significance of recalcitrant emotions (or anti-quasijudgmentalism). Royal Institute of Philosophy Supplements, 52, 127-145.

Deonna, J. y Teroni, F. (2012). The emotions: A philosophical introduction. Nueva York: Routledge.

Doris, J. (2002). Lack of Character: Personality and Moral Behavior. Cambridge: Cambridge University Press.

Fleming, J. y Ledogar, R. (2008). Resilience, an evolving concept: A review of literature relevant to aboriginal research. Pimatisiwin, 6 (2), 7-23.

Goldie, P. (2012). The mess inside: Narrative, emotion, $\mathcal{E}$ the mind. Oxford: Oxford University Press.

Goldie, P. (2000). The emotions: A philosophical exploration. Oxford: Oxford University Press.

Hansberg, O. (1996). La diversidad de las emociones. México: Fondo de Cultura Económica.

Harman, G. (1999). Moral philosophy meets social psychology: Virtue ethics and the fundamental attribution error. Proceedings of the Aristotelian Society, 99, 315-331.

Harman, G. (2000). The nonexistence of character traits. Proceedings of the Aristotelian Society, 100, 223-226.

Homiak, M. (Otoño de 2016). Moral character. E. N. Zalta (ed.) Recuperado el 12 de enero de 2019, de The Stanford Encyclopedia of Philosophy: 
https://plato.stanford.edu/archives/fall2016/entries/mo ral-character/

Krashin, D., Murinova, N., Howe, C. Q. y Ballantyne, J. (2014). Biology of suffering. R. M. Green, \& N. J. Palpant (eds.) Recuperado el 24 de abril de 2017, de Suffering and bioethics:

DOI:10.1093/acprof:oso/9780199926176.003.0006

Roth, J. (2017 [1930]). Job: Historia de un hombre sencillo (5a ed.).

(B. Vias Mahou, Trad.) Barcelona: Acantilado.

Russell, D. (2009). Practical intelligence and the virtues. Oxford: Clarendon Press.

Sacks, O. (2009 [1985]). El hombre que confundió a su mujer con un sombrero, J. M. Álvarez Flórez (trad.) México: Anagrama-Colofón.

Snow, N. (2015). Models of virtue. En L. Besser-Jones, \& M. Slote, The Routledge Companion to Virtue Ethics (págs. 359-373). Nueva York: Routledge.

Snow, N. (2010). Virtue as social intelligence: An empirically grounded theory. Nueva York: Routledge.

Stump, E. (2010). Wandering in darkness: Narrative and the problem os suffering. Oxford: Oxford University Press.

Tappolet, C. (2012). Emotions, perceptions and emotional illusions. En C. Calabi (ed.), Perceptual Illusions (págs. 205-222). London: Palgrave Macmillan.

Vranas, P. (2005). The indeterminacy paradox: Character evaluations and human psychology. Noûs, 39, 1-42. 